\title{
Analysis of human meiotic recombination events with a parent-sibling tracing approach
}

Yun-Shien Lee ${ }^{1,2 \dagger}$, Angel Chao ${ }^{3 \dagger}$, Chun-Houh Chen ${ }^{4}$, Tina Chou ${ }^{4}$, Shih-Yee Mimi Wang ${ }^{5}$ and Tzu-Hao Wang ${ }^{2,3^{*}}$

\begin{abstract}
Background: Meiotic recombination ensures that each child inherits distinct genetic materials from each parent, but the distribution of crossovers along meiotic chromosomes remains difficult to identify. In this study, we developed a parent-sibling tracing (PST) approach from previously reported methods to identify meiotic crossover sites of GEO GSE6754 data set. This approach requires only the single nucleotide polymorphism (SNP) data of the pedigrees of both parents and at least two of children.

Results: Compared to other SNP-based algorithms (identity by descent or pediSNP), fewer uninformative SNPS were derived with the use of PST. Analysis of a GEO GSE6754 data set containing 2,145 maternal and paternal meiotic events revealed that the pattern and distribution of paternal and maternal recombination sites vary along the chromosomes. Lower crossover rates near the centromeres were more prominent in males than in females. Based on analysis of repetitive sequences, we also showed that recombination hotspots are positively correlated with SINE/MIR repetitive elements and negatively correlated with LINE/L1 elements. The number of meiotic recombination events was positively correlated with the number of shorter tandem repeat sequences.

Conclusions: The advantages of the PST approach include the ability to use only two-generation pedigrees with two siblings and the ability to perform gender-specific analyses of repetitive elements and tandem repeat sequences while including fewer uninformative SNP regions in the results.
\end{abstract}

\section{Background}

Meiotic recombination is important for generating genetic diversity. Meiotic recombination occurs between homologous chromosomes during chiasmata formation, a process that is required for normal chromosomal segregation during meiosis. While variation in recombination rates is a ubiquitous feature of the human genome [1], the mechanisms governing the distribution of crossovers along meiotic chromosomes remain largely unclear, with the exception of the recent discovery that $\operatorname{Prdm} 9$ is involved in the activation of mammalian recombination hotspots [2-5]. Sex-specific effects [6-8] on regional meiotic recombination have been described. Recombination rates are approximately 1.7 -fold higher in female meiosis than in male meiosis. In addition, crossover rates in males are 5-fold lower near

\footnotetext{
* Correspondence: knoxtn@cgmh.org.tw

† Contributed equally

${ }^{2}$ Genomic Medicine Research Core Laboratory (GMRCL), Chang Gung

Memorial Hospital, Tao-Yuan, Taiwan

Full list of author information is available at the end of the article
}

centromeres but 10 -fold higher near telomeres compared with those in females [9]. These differences could be related to sex-specific patterns of initiation of synapses between homologs. For example, synaptonemal complex lengths are shorter in males than in females [10], and synapses appear preferentially in subtelomeric regions in males [11].

Meiotic recombination events can be measured directly or indirectly [12]. Physical crossovers between homologous chromosomes, indicating meiotic recombination events, can be directly observed at specific time points during spermatogenesis [13]. Alternatively, crossovers may be analyzed directly in cytogenetic analysis by labeling meiosis-related proteins, such as MLH1 [14]. Despite the unequivocal value of direct analysis, these techniques are labor-intensive and precision is limited. Therefore, most analyses of human recombination currently rely on indirect approaches such as genetic linkage analysis of human pedigrees. This involves tracking the inheritance of alleles at multiple polymorphic markers (short tandem repeat polymorphisms, STRP; or
C Biomed Central

(c) 2011 Lee et al; licensee BioMed Central Ltd. This is an Open Access article distributed under the terms of the Creative Commons Attribution License (http://creativecommons.org/licenses/by/2.0), which permits unrestricted use, distribution, and reproduction in any medium, provided the original work is properly cited. 
single nucleotide polymorphisms, SNP) along the chromosomes across generations [15-17].

Molecular markers in individuals with known pedigrees can be traced to an ancestral identity using either the identity by descent (IBD) method [12] or the identity by state (IBS) method [18]. Two alleles at a particular locus in the progeny are assumed to be identical if they are derived from an identical locus in a common ancestor. The IBD method requires knowledge of the genotypes of three generations to determine if the DNA segments are identical by descent from each generation. In the IBD method, shared results between each child and his/her paternal and maternal grandparents are analyzed separately. A paternal recombination event is detected when the IBD sharing "switches" from one paternal grandparent to the other. This application can be applied in the same manner for the maternal side. For instance, meiotic events can be switched between 2 SNP sites (Figure 1A and Additional File 1A). Therefore, application of the IBD method requires the pedigrees of three generations [12]. The IBS method was used to detect meiotic recombination sites between individuals by analyzing allele sharing between siblings [18].
Recently, Ting et al. also proposed another method for identifying meiotic recombination patterns based on two-generation pedigrees (pediSNP) [19]. In the pediSNP method, genotypes of two children are analyzed and compared with the genotype of one parent [19].

Based on the distribution of SNPs in both parents and multiple siblings, meiotic cross sites in human chromosomes can be identified. This method was first proposed by Coop et al. in 2008 to trace the "informative markers" transmitted by the father to each offspring [6]. They defined the "informative markers" as SNPs that are heterozygous in the father and homozygous in the mother. In 2009, Chowdhury et al. used two datasets, namely, the Autism Genetic Research Exchange (AGRE) and the Framingham Heart Study (FHS), to characterize the variation in recombination phenotypes [20]. They analyzed sex differences and recombination jungles across the human genome, and described the gene loci associated with recombination phenotypes [20].

In this study, we have used a parent-sibling tracing (PST) approach, which was derived from two previous reports [6,20], to analyze the Genomic Medicine Research Core Laboratory, Taiwan (GMRCL) dataset of

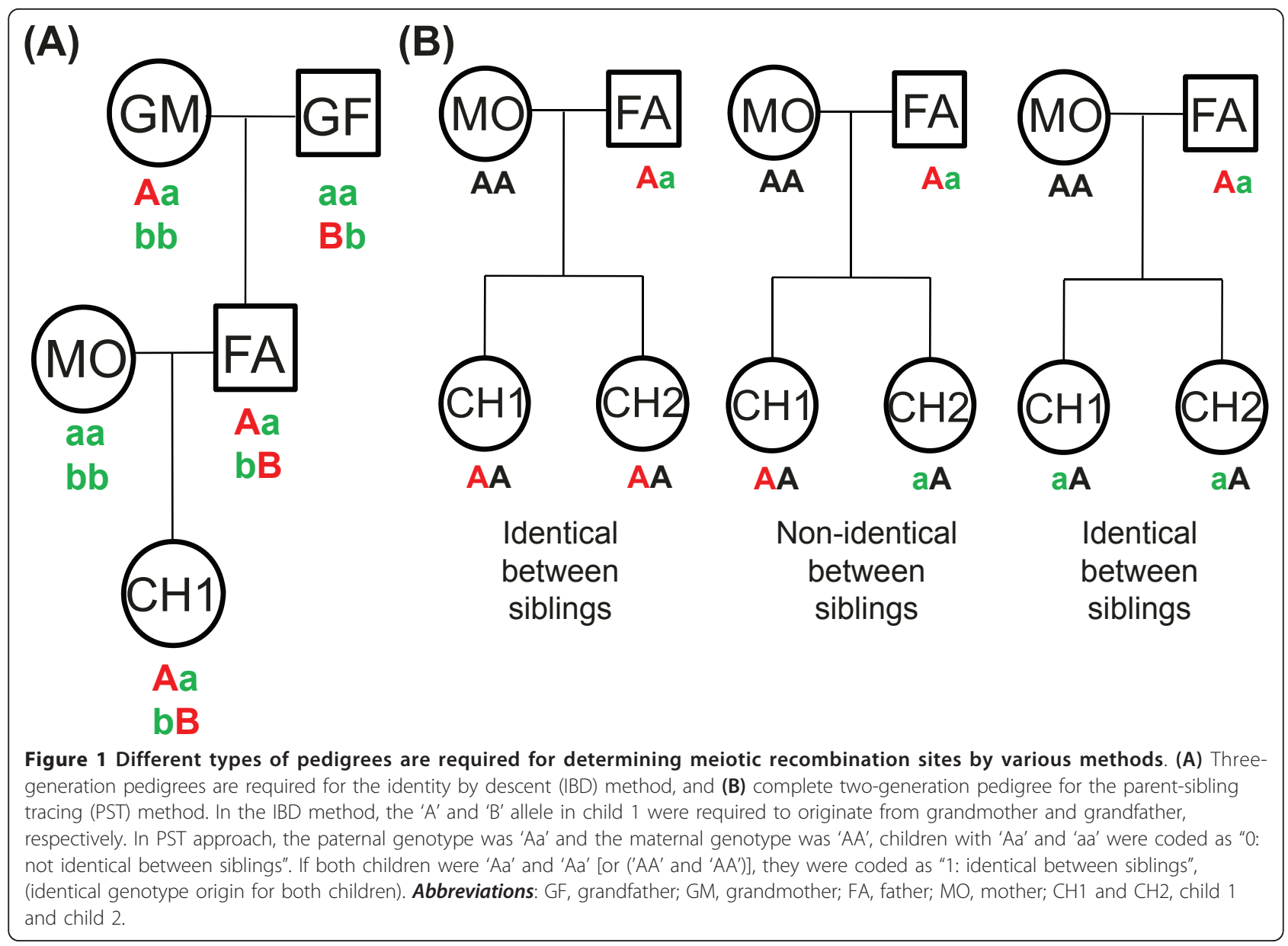


Affymetrix SNP6.0 arrays which consists of $900 \mathrm{~K}$ SNP markers and the GSE6754 dataset from Gene Expression Omnibus (GEO) [21], which consists of 853 families. Our analyses of this dataset of 2,145 meioses resulted in a 1-Mb-resolution recombination map. In addition, we were able to characterize the relationships between recombination sites and repetitive elements as well as the relationships between recombination sites and tandem repeats sequences.

\section{Results}

\section{Comparison of two methods of detecting meiotic recombination sites}

We used the GMRCL dataset of $900 \mathrm{~K}$ SNPs as a reference standard for comparison between the PST approach (Figure 1B) and previous approaches such as the IBD method [12] (Figure 1A). The code calling schema of PST is depicted in Figure 1B and Additional File 1B. Using chromosome 1 as an example, IBD analysis in both children could define the sites of meiotic recombination for paternal gametes. In child 1 and child 2, we observed 1 and 4 meiotic recombination events on their paternal gametes, respectively (Figures $2 \mathrm{~A}$ and $2 \mathrm{~B}$ ). Using the PST approach, we could analyze the paternal genotypes for both children. When the paternal genotype was Aa and the maternal genotype was AA, children with Aa and AA were coded as "0: not identical between siblings". If both children were Aa and Aa [or (AA and AA)], they were coded as "1: identical between siblings" (identical genotype origin for both children). The PST approach (Figure $2 \mathrm{C})$ detected the recombination sites of the combinatorial results for child 1 and child 2 as determined by IBD (Figures $2 \mathrm{~A}$ and $2 \mathrm{~B}$ ). These results indicate that, using the SNP information of only two generations, PST can identify the origin of the recombination site. For the IBD method, information from three generations is required to determine whether the origin is from the grandfather or the grandmother. The 43 recombination sites identified in the GMRCL dataset using the IBD and PST methods are shown in Additional file 2.

Comparison of the code calling schemas between the IBD and PST methods showed that IBD identified fewer genotyping combination calls than the PST approach. For instance, when we analyzed the recombination sites in the $100-\mathrm{kb}$ genomic region located at $114.6 \mathrm{Mb}$ on chromosome 1 (Figures $2 \mathrm{~B}$ and $2 \mathrm{C}$, indicated with the arrow), the numbers of uninformative SNPs in the recombination site for the IBD and PST methods were 22 and 19, respectively (Figures $2 \mathrm{D}$ and $2 \mathrm{E}$ ), resulting in uninformative regions of $54 \mathrm{~kb}$ for the IBD method (Figure 2D) and $48 \mathrm{~kb}$ for the PST approach (Figure 2E), respectively.

The use of the IBD and PST methods in the GMRCL sample led to the identification of 43 paternal

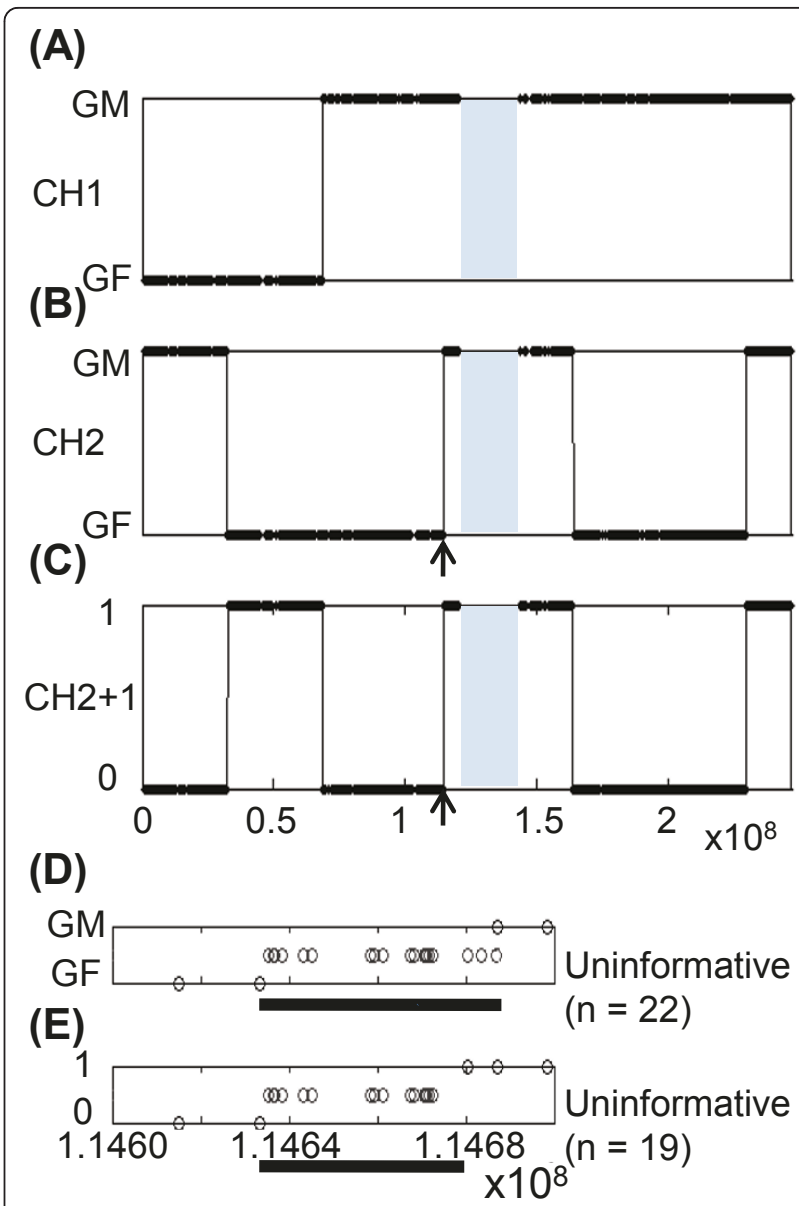

Figure 2 The paternal recombination site on chromosome 1 of child 1 and 2 ( $\mathrm{CH} 1$ and $\mathrm{CH} 2$, defined in Figure 1) in the GMRCL dataset were defined using the identity by descent (IBD) (A, B, D) and parent-sibling tracing (PST) (C, E) methods. The grandmother and grandfather origin of paternal recombination is indicated as GM and GF, respectively. Children with identical or not identical origin are indicated as 1 and 0 , respectively. Panels $\mathbf{D}$ and $\mathbf{E}$ are the enlarged view of the 114.6 -114.7 kb region on chromosome 1 shown in panels $\mathbf{B}$ and $\mathbf{C}$, respectively, which are indicated by the black arrows. D and E: the SNP sites (open circles) that could not be mapped to either GF or GM in the IBD method, or to either an identical or non-identical status using the PST approach, are indicated as a uninformative SNPs. The calling schema of IBD and PST methods is shown in Additional File 1. The chromosomal regions without any SNP site in the Affymetrix Genome-Wide Human SNP 6.0 arrays are marked as gray blocks (A to $\mathbf{C}$ ).

recombination sites in child 1 and child 2 . The mean numbers of uninformative SNP for the 43 paternal recombination sites were 71.2 and 36.7 for the IBD and PST methods, respectively (Table 1 ). The mean sizes of the uninformative regions for the 43 paternal recombination sites were $253 \pm 349 \mathrm{~kb}$ (mean \pm SD) with 110 (58 - 336) in Q2 (Q1-Q3) for the IBD method, and 167 $\pm 391 \mathrm{~kb}$ with $60(23-157)$ in Q2 (Q1-Q3) for the PST approach (Table 1$)$. The paired t-test showed that the 
Table 1 Comparison of the size and SNP numbers in uninformative regions

\begin{tabular}{|c|c|c|c|c|c|}
\hline & \multirow[b]{2}{*}{ Sibling\# } & \multicolumn{2}{|c|}{ IBD } & \multicolumn{2}{|c|}{ PST } \\
\hline & & Q2 (Q1 - Q3) kb ${ }^{\dagger}$ & SNP\#* & Q2 (Q1 - Q3) kb ${ }^{\dagger}$ & SNP\#* \\
\hline \multicolumn{6}{|l|}{$900 \mathrm{~K}$} \\
\hline Paternal & 2 & $110(58-336)$ & 71.2 & $60(23-157)$ & 36.7 \\
\hline Maternal & 2 & & & $61(19-189)$ & 39.6 \\
\hline \multicolumn{6}{|c|}{ Autism_3117 } \\
\hline Paternal & 4 & $3291(2255-5738)$ & 12.31 & $1751(1270-3347)$ & 7.1 \\
\hline Maternal & 4 & $2683(1249-5796)$ & 15.34 & 1806 (947 - 3389) & 5.9 \\
\hline \multicolumn{6}{|c|}{ Autism_3180 } \\
\hline Paternal & 6 & $3768(1858-6420)$ & 16.1 & $1701(938-2853)$ & 5.8 \\
\hline Maternal & 6 & $2842(1145-5789)$ & 14.6 & $2151(1234-3712)$ & 6.9 \\
\hline \multicolumn{6}{|c|}{ Autism_8071 } \\
\hline Paternal & 4 & $3557(1877-6415)$ & 15.6 & $1892(1195-3230)$ & 7.5 \\
\hline Maternal & 4 & & & $2046(1130-4031)$ & 7.8 \\
\hline
\end{tabular}

† Q2 (Q1 - Q3): Q2 (second quartile) = 50th percentile; Q1 (first quartile) = 25th percentile; Q3 (third quartile) = 75th percentile

* SNP\#: Average number of SNPs in the "uninformative" region

PST approach resulted in significantly shorter uninformative regions than the IBD method $\left(\mathrm{P}<10^{-10}\right)$.

\section{Analysis of the GEO dataset GSE6754 containing 11,000 SNP markers}

The Affymetrix Human Mapping 10 K 2.0 Arrays (containing $10 \mathrm{~K} \mathrm{SNPs)} \mathrm{were} \mathrm{used} \mathrm{to} \mathrm{map} \mathrm{autism} \mathrm{suscept-}$ ibility loci in the GSE6754 dataset [22]. Three threegeneration pedigrees (family ID: $3117,3180,8071$ ) were selected to compare the usefulness of the IBD and PST methods. Since the $10 \mathrm{~K} 2.0$ array covered fewer SNPs, the mean size of uninformative regions were about 20fold higher and the number of uninformative SNPs was approximately 6-fold lower than those of SNP 6.0 Arrays. Compared to other approaches, the PST approach identified fewer uninformative SNPs and smaller uninformative genomic regions (Table 1).

In the 3864 arrays (853 families, 1721 parents, 2145 siblings) analyzed using the PST approach, the mean number of maternal recombination events was approximately 1.67-fold higher than that of paternal origin, with the highest value observed on chromosome 17 (2.00fold) and the lowest on chromosome 22 (1.32-fold) (Table 2). The distribution of recombination events of paternal origin (mean $23.8 \pm 4.1$, median 22.5) and maternal origin (mean $39.5 \pm 5.7$, median 38.0 ) is presented in Figure 3A. The numbers of recombination events of each chromosome (2,145 maternal and paternal meioses) are summarized in Table 2.

In order to identify the regions with the highest and the lowest number of recombination events, we scanned the entire human genome. We first divided the genome into 2,765 bins of 1-Mb each. We then identified the number of recombination sites in each bin separately for female and male meioses. The results obtained from chromosome 1 are shown in Figure 3B (see the Additional file 3 for the results on other chromosomes). We also compared the recombination maps obtained from dataset GSE6754 with Marshfield map [23] (Figure 3B, middle panel), and Icelandic map [16] (Figure 3B, lower panel). The correlation coefficients between the data in

Table 2 Number of recombination sites in 2145 siblings from 853 families

\begin{tabular}{lccr}
\hline Chromosome & Male & Female & Female/male \\
\hline chr1 & 3990 & 6819 & 1.71 \\
chr2 & 3917 & 6723 & 1.72 \\
chr3 & 3507 & 5847 & 1.67 \\
chr4 & 3007 & 5361 & 1.78 \\
chr5 & 2864 & 5255 & $1.83^{*}$ \\
chr6 & 2971 & 5063 & 1.70 \\
chr7 & 2582 & 4560 & 1.77 \\
chr8 & 2378 & 4212 & 1.77 \\
chr9 & 2495 & 3883 & 1.56 \\
chr10 & 2544 & 4417 & 1.74 \\
chr11 & 2348 & 4017 & 1.71 \\
chr12 & 2503 & 4140 & 1.65 \\
chr13 & 1996 & 3162 & 1.58 \\
chr14 & 2007 & 2784 & $1.39^{*}$ \\
chr15 & 1988 & 2859 & $1.44^{*}$ \\
chr16 & 1762 & 2902 & 1.65 \\
chr17 & 1393 & 2783 & $\mathbf{2 . 0 0}$ \\
chr18 & 1856 & 2903 & 1.56 \\
chr19 & 1210 & 2072 & 1.71 \\
chr20 & 1612 & 2383 & $1.48^{*}$ \\
chr21 & 1004 & 1444 & $1.44^{*}$ \\
chr22 & 965 & 1265 & $\mathbf{1 . 3 1 *}$ \\
chrx & & 2932 & \\
\hline P & & & \\
\hline
\end{tabular}

* $P$ value $<0.01$ (chi square analysis under the null hypothesis that the maleto-female proportion was 1.667 ) 


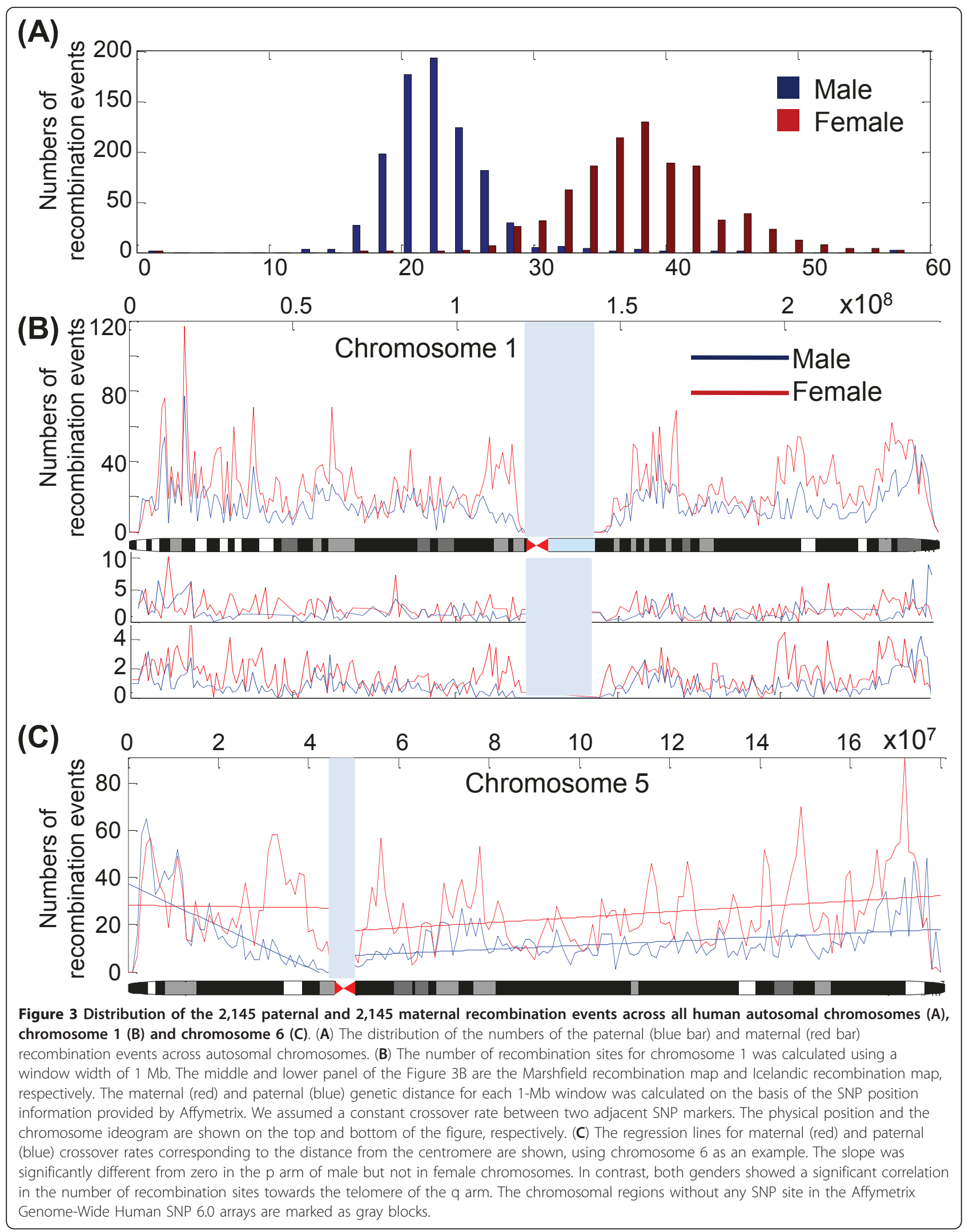


GSE6754 map and Icelandic map and Marshfield map were $r=0.49$ and $r=0.31$, respectively.

To test the hypothesis that recombination rates are lower near the centromere but higher near the telomeres in men, we analyzed the correlation between the distances from the recombination sites to the centromere and the number of recombination sites. We found significant correlations $(\mathrm{P}<0.00001)$ on chromosomes $1 \mathrm{q}, 2 \mathrm{p}, 3 \mathrm{q}, 4 \mathrm{q}, 5 \mathrm{p}, 5 \mathrm{q}, 6 \mathrm{p}, 6 \mathrm{q}, 7 \mathrm{q}, 8 \mathrm{q}, 9 \mathrm{p}, 9 \mathrm{q}, 10 \mathrm{p}, 10 \mathrm{q}$, $11 \mathrm{q}, 12 \mathrm{p}, 12 \mathrm{q}, 16 \mathrm{q}, 18 \mathrm{q}, 19 \mathrm{q}, 20 \mathrm{q}, 21 \mathrm{q}$ in men. In contrast, similar correlations were found only on chromosome $1 \mathrm{q}$ and $6 \mathrm{q}$ in women (Table 3 ). For instance, the slope of correlation was significant in $\mathrm{p}$ arm of chromosome 5 in men but not in women (Figure 3C). On the other hand, both sexes showed significant correlations in the number of recombination sites near the telomere in the $\mathrm{q}$ arm. SNP information was not available for the p arm of chromosomes 13, 14, 15, 21, and 22 .

\section{Relation between the recombination site and repetitive elements}

We compiled 57 major repetitive element classes that were characterized by RepeatMasker [24]. Twenty-three

\section{Table 3 Correlation of the distance from the recombination site to the centromere with the number of} recombination events

\begin{tabular}{|c|c|c|c|c|}
\hline \multirow[b]{2}{*}{ Chromosome } & \multicolumn{2}{|c|}{ Male } & \multicolumn{2}{|c|}{ Female } \\
\hline & $\mathrm{p}$ arm & q arm & $\mathrm{p}$ arm & q arm \\
\hline chr1 & 0.03979 & 4.1E-10 & 0.63413 & 3.7E-10 \\
\hline chr2 & 3.1E-07 & 0.12 & 0.00783 & 0.01009 \\
\hline chr3 & 0.00127 & $1.1 \mathrm{E}-11$ & 0.00022 & 3.9E-05 \\
\hline chr4 & 0.00865 & 2.1E-09 & 0.20608 & 0.00063 \\
\hline chr5 & $1.9 \mathrm{E}-07$ & $5.3 \mathrm{E}-06$ & 0.87512 & 0.00262 \\
\hline chr6 & $3.4 \mathrm{E}-07$ & 6.7E-16 & 0.10238 & 5.7E-08 \\
\hline chr7 & 0.00329 & $2.2 \mathrm{E}-07$ & 0.9658 & 0.00189 \\
\hline chr8 & 3.9E-05 & $2.6 \mathrm{E}-06$ & 0.33193 & 0.41184 \\
\hline chr9 & $2.3 \mathrm{E}-12$ & $1.9 \mathrm{E}-08$ & 0.00064 & 0.01546 \\
\hline chr10 & 2.3E-09 & $1.6 \mathrm{E}-07$ & 0.92443 & 0.00077 \\
\hline chr11 & 0.01294 & 1.1E-08 & 0.83831 & $4 \mathrm{E}-05$ \\
\hline chr12 & $6.2 \mathrm{E}-06$ & $3.6 \mathrm{E}-06$ & 0.17744 & 0.00675 \\
\hline chr13 & NA & 0.88298 & NA & 0.41116 \\
\hline chr14 & NA & 0.42025 & NA & 0.0348 \\
\hline chr15 & NA & 0.10395 & NA & 0.00605 \\
\hline chr16 & 0.09796 & $6.8 \mathrm{E}-09$ & 0.88747 & 0.34803 \\
\hline chr17 & 0.7478 & 0.00062 & 0.15536 & 0.69596 \\
\hline chr18 & 0.02079 & $2 \mathrm{E}-09$ & 0.3442 & 0.22101 \\
\hline chr19 & 0.95524 & 4.7E-10 & 0.06423 & 0.11279 \\
\hline chr20 & 4.1E-05 & $2.6 \mathrm{E}-07$ & 0.33546 & 0.04724 \\
\hline chr21 & NA & $2.3 \mathrm{E}-10$ & NA & 0.66508 \\
\hline chr22 & NA & 0.0005 & NA & 0.05598 \\
\hline
\end{tabular}

*NA: SNP information not available for the $\mathrm{p}$ arm of chromosomes 13, 14, 15, 21 , and 22.

The values in bold indicate a $\mathrm{P}$ value $<1 \mathrm{E5}$ repetitive-element classes were identified in more than 6,000 sites in the human genome. After downloading the location information of the human $\mathrm{CpG}$ islands from the UCSC database [25], we divided the genome into 2,765 bins of $1-\mathrm{Mb}$ each and determined the number of repetitive-element sites in each bin. Using the 53,487 repetitive-elements on chromosome 1 as an example, we depicted the distribution of SINE/MIR (green lines in Figure 4A) and LINE/L1 sites (green lines in Figure 4C). In addition, the distributions of meiotic recombination sites (both paternal and maternal combined) are shown as blue lines. In each $1-\mathrm{Mb}$ bin, we also analyzed the correlation between the number of meiotic recombination sites and the number of SINE/ MIR (plotted in Figure 4B) and LINE/L1 sites (plotted in Figure 4D). The correlation coefficients between recombination sites and SINE/MIR and the correlation coefficients between recombination sites and LINE/L1 were $0.23(\mathrm{P}=0.0005)$ and $0.29(\mathrm{P}=0.00001)$, respectively.

The correlation coefficients and the corresponding $\mathrm{P}$ values for each of the 23 repetitive-elements, CpG island sites, and meiotic recombination sites are summarized in Table 4. The repetitive elements SINE/MIR, DNA/hATCharlie, DNA/hAT, LINE/L2, SINE/Alu, DNA/hATTip100, DNA/hAT-Blackjack were positively correlated with meiotic recombination sites. In contrast, repetitive elements, which included LINE/L1, LTR/ERVK, and Low complexity (Table 4), showed negative correlation with meiotic recombination sites. In general, we found no significant differences in the distribution of maternal and paternal recombination sites. The scatter plots of the correlation analyses of repetitive elements SINE/ MIR and LINE/L1 in the entire human genome are shown in Figure 5.

\section{Relation between recombination sites and the length of tandem repeat sequences}

Repetitive elements, including tandem repeat sequences, are distributed widely throughout the genome. Tandem DNA repeats are defined as a repeated pattern of two or more nucleotides. The pattern can range in length from 2 to $\sim 100$ base pairs (bp) (for example (CATG)n in a genomic region) [26]. In this study, a total 947,696 tandem repeats sequences were identified using the Tandem Repeats Finder [26]. The length distribution of the tandem repeats are shown in Figure 6A, where the 25, 50 and 75 percentile of the length of the tandem repeats were 4,15 and $24 \mathrm{bp}$, respectively.

We divided the genome into 2,765 bins of $1-\mathrm{Mb}$ each and determined the number of tandem repeats in each bin. We then analyzed the correlation between the number of maternal meiotic recombination sites and the number of tandem repeats (Figure 6B); the correlation 


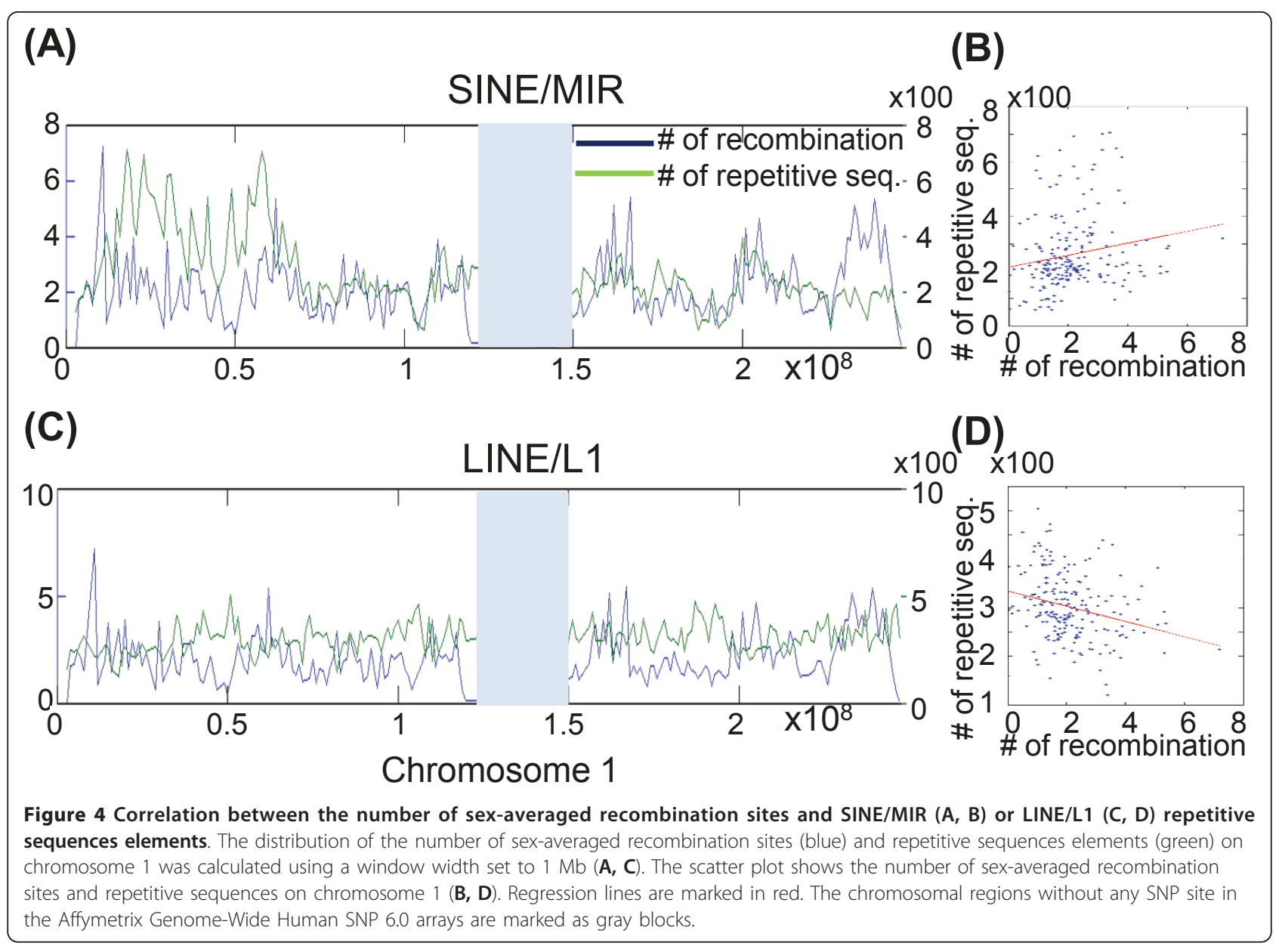

coefficient was $0.11\left(\mathrm{P}<2 \times 10^{-7}\right)$. Furthermore, we grouped tandem repeats into 4 quartiles by the length of these repeat sequences, as (Q1) 1-4, (Q2) 5-15, (Q3) 1624 and $(\mathrm{Q} 4)>25 \mathrm{bp}$. The correlation coefficients between recombination sites and the 4 quartiles were $0.25\left(\mathrm{P}<1 \times 10^{-16}\right), 0.11\left(\mathrm{P}<2 \times 10^{-8}\right), 0.04(\mathrm{P}=0.08)$ and $0.03(\mathrm{P}=0.16)$, respectively (Figures $6 \mathrm{C}-\mathrm{F})$. These results showed that the maternal meiotic recombination sites were positively correlated with shorter repeat sequences and less correlated with longer repeat sequences. Similarly, we analyzed the correlation between the number of paternal meiotic recombination sites and the number of tandem repeats, with $r=0.12$ $\left(\mathrm{P}<5 \times 10^{-9}\right)$. The correlation coefficients for the 4 subgroups were $0.19\left(\mathrm{P}<1 \times 10^{-16}\right), 0.09\left(\mathrm{P}<4 \times 10^{-6}\right)$, $0.09\left(\mathrm{P}<3 \times 10^{-6}\right)$ and $0.05(\mathrm{P}=0.004)$, respectively (Additional file 4).

\section{Discussion}

In this study, we use a PST approach to analyze the sites of meiotic recombination in two-generation pedigrees. We first tested it on a GMRCL dataset of the
Affymetrix SNP 6.0 array consisting of $900 \mathrm{~K}$ SNP markers, followed by a $10 \mathrm{~K}$ GSE6754 dataset. In the GSE6754 dataset, which was previously used for mapping autism risk loci, most data are based on two-generation pedigrees (1,168 families) as this dataset contains only 29 three-generation pedigrees. Although the PST approach requires only pedigrees of two generations, it requires information from at least two siblings. The use of SNPs as genetic markers to identify recombination sites can often result in the inclusion of uninformative regions. However, the size of uninformative regions that result from the PST approach is significantly lower than that seen from the use of the IBD method (Table 1).

We next assessed whether crossovers may alter the DNA sequence by causing de novo mutations at sites of recombination. Given that the uninformative regions of PST were relatively small, eight recombination events were identified with sizes of less than $2 \mathrm{~kb}$. Notably, we did not identify any sequence variation at these recombination points (data not shown). This observation needs further validation by sequencing more datasets. 
Table 4 Correlation between the recombination sites and particular repeats

\begin{tabular}{|c|c|c|c|c|c|c|c|}
\hline & & Pater & & Materr & & Both & \\
\hline Repeat & Number & Corr & $P$ & Corr. & $P$ & Corr. & $\mathbf{P}$ \\
\hline SINE/MIR & 510580 & 0.22 & $1 E-16$ & 0.30 & $1 E-16$ & 0.29 & $1 \mathrm{E}-16$ \\
\hline DNA/hAT-Charlie & 214901 & 0.18 & $1 E-16$ & 0.31 & $1 E-16$ & 0.29 & $1 \mathrm{E}-16$ \\
\hline DNA/hAT & 10624 & 0.16 & $3 E-13$ & 0.24 & $1 \mathrm{E}-16$ & 0.23 & $1 \mathrm{E}-16$ \\
\hline LINE/L2 & 397294 & 0.16 & $3 \mathrm{E}-13$ & 0.22 & $1 \mathrm{E}-16$ & 0.21 & $1 \mathrm{E}-16$ \\
\hline SINE/Alu & 926299 & 0.10 & $1 \mathrm{E}-04$ & 0.22 & $1 \mathrm{E}-16$ & 0.19 & $1 \mathrm{E}-16$ \\
\hline DNA/hAT-Tip100 & 26087 & 0.10 & $3 \mathrm{E}-05$ & 0.20 & $1 \mathrm{E}-16$ & 0.18 & $1 \mathrm{E}-16$ \\
\hline DNA/hAT-Blackjack & 17019 & 0.15 & $4 \mathrm{E}-12$ & 0.16 & $2 \mathrm{E}-13$ & 0.17 & $1 \mathrm{E}-16$ \\
\hline Simple_repeat & 343474 & 0.19 & $1 E-16$ & 0.11 & $4 \mathrm{E}-06$ & 0.16 & $8 \mathrm{E}-14$ \\
\hline LINE/CR1 & 52244 & 0.09 & $4 \mathrm{E}-04$ & 0.15 & $5 \mathrm{E}-12$ & 0.13 & $9 \mathrm{E}-09$ \\
\hline DNA/TcMar-Tc2 & 6979 & 0.11 & $1 \mathrm{E}-05$ & 0.13 & $3 \mathrm{E}-08$ & 0.13 & $3 \mathrm{E}-08$ \\
\hline DNA/TcMar-Mariner & 14046 & 0.10 & $5 \mathrm{E}-05$ & 0.13 & $2 \mathrm{E}-08$ & 0.12 & $2 \mathrm{E}-07$ \\
\hline $\mathrm{CpG}$ & 19661 & 0.06 & $2 \mathrm{E}-01$ & 0.10 & $3 \mathrm{E}-05$ & 0.11 & $1 \mathrm{E}-06$ \\
\hline LTR/ERVL-MaLR & 292520 & 0.11 & $2 \mathrm{E}-06$ & 0.09 & $6 \mathrm{E}-04$ & 0.11 & $8 \mathrm{E}-06$ \\
\hline LTR/Gypsy? & 6912 & 0.05 & 1 & 0.11 & $4 \mathrm{E}-06$ & 0.08 & $2 \mathrm{E}-03$ \\
\hline Unknown & 6174 & 0.05 & $5 E-01$ & 0.06 & $4 \mathrm{E}-01$ & 0.05 & 1 \\
\hline LINE/RTE & 15421 & 0.04 & 1 & 0.06 & $2 \mathrm{E}-01$ & 0.05 & 1 \\
\hline LTR/Gypsy & 9429 & 0.02 & 1 & 0.04 & 1 & 0.03 & 1 \\
\hline DNA/TcMar-Tigger & 87328 & -0.01 & 1 & 0.04 & 1 & 0.01 & 1 \\
\hline LTR/ERVL & 134989 & -0.03 & 1 & -0.03 & 1 & -0.04 & 1 \\
\hline LTR/ERV1 & 139204 & -0.13 & 7E-09 & -0.10 & $6 \mathrm{E}-05$ & -0.13 & $2 \mathrm{E}-08$ \\
\hline Low_complexity & 314872 & -0.11 & $3 E-06$ & -0.17 & $6 \mathrm{E}-15$ & -0.18 & $1 \mathrm{E}-16$ \\
\hline LTR/ERVK & 8019 & -0.19 & $1 E-16$ & -0.16 & $3 E-13$ & -0.19 & $1 \mathrm{E}-16$ \\
\hline LINE/L1 & 767428 & -0.16 & $8 \mathrm{E}-13$ & -0.19 & $1 \mathrm{E}-16$ & -0.20 & $1 \mathrm{E}-16$ \\
\hline
\end{tabular}

1. Repeat classes including more than 6000 repeats were considered for the purpose of analyses.

2. Corr.: correlation coefficients between the recombination sites and specific repeats.

3. $\mathrm{P}$ values under the null hypothesis of an absence of correlation. The Bonferroni's correction was applied for multiple comparisons. An adjusted $\mathrm{P}$ value $>1$ was reported as 1.

4. The values in bold indicate a P value $<1 \mathrm{E} 5$

The average number of recombination events observed with the PST approach was similar to the findings of other studies. The distribution of recombination events showed a mean value of 23.8 in paternal origin and 39.5 in maternal origin. Chowdhury et al reported the genome-wide recombination events in paternal origin ranged from 25.9 to 27.3 while in maternal origin ranged from 38.4 to 47.2 [20]. Another study by Cheung et al demonstrated that the mean numbers of recombination events were 24.0 in male meiosis and 38.4 in female meiosis [15].

In an indirect pedigree analysis using SNPs as genetic markers, Cheung et al [15] reported that several recombination events appeared to occur nearer to the telomeres. Using the PST approach, we analyzed the distance between the recombination site and the centromere for each gender separately (Table 3 ). In male meiosis, most of the crossovers are located in the $\mathrm{q}$ arms, and the number of recombination events increased significantly when moving from centromeres to telomeres. Interestingly, we observed fewer recombination events in the $\mathrm{p}$ arms of female chromosomes, resulting in the male-to-female ratio of 1.67 (Table 2). In women, only chromosomes 1q and $6 \mathrm{q}$ showed a significant, positive correlation between the number of recombination sites and distance from the centromere (Table 3).

To determine the extensive sequence-context variation in recombination hotspots, Myers et al. constructed a fine-scale map of recombination rates and hotspots across the human genome based on genotypes of 1.6 million SNPs in three sample populations, including 24 European Americans, 23 African Americans, and 24 Han Chinese [27]. The authors reported an increase of recombination hotspots in the regions surrounding coding genes, though these were preferentially located outside the transcribed regions. The analysis of the relationships between recombination hotspots and repeat elements indicated that L2 and THE1B are unusually high in hotspots, whereas L1 elements are low [27]. In this study, we identified a similar pattern of frequent hotspots in L2 as opposed to the low number of hotspots in L1 elements (Table 4). Of note, results showed that the majority of the hotspots in both paternal and maternal meioses were similar. 


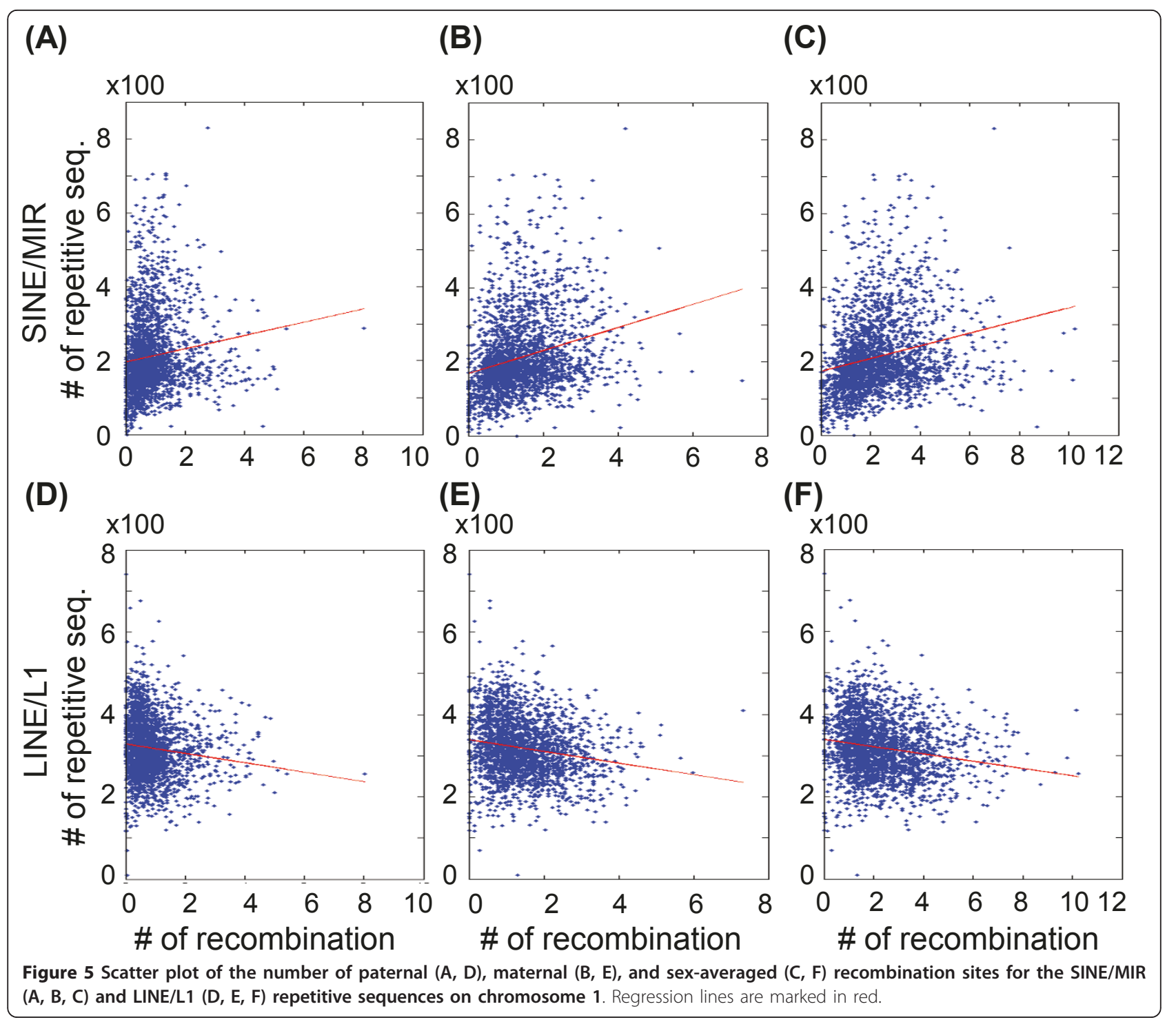

\section{Conclusion}

Human chromosomes are characterized by prominent differences in the pattern and rate of meiotic recombination events. Significant inter-individual and gender differences also exist. The major advantages of the PST approach include the use of two-generation pedigrees with two or more siblings, fewer uninformative SNP regions, and the ability to perform gender-specific analyses of recombination hotspots (using databases derived from high density arrays such as Affymetrix SNP6.0) and repetitive elements. An accurate determination of meiotic crossovers using this approach may prove useful to explore the biology of human chromosomes.

\section{Methods}

Identification of meiotic recombination sites

In the present study we compared different SNP-based methods for detecting recombination points, i.e. IBD (Figure 1A) [12], and PST (Figure 1B). The code calling schema for the IBD and PST methods are depicted in the Additional Files $1 \mathrm{~A}$ and $1 \mathrm{~B}$. The meiosis recombination sites were exported from the PSTReader, a MATLAB-based program (version 7.9). The PSTReader was used to define the recombination sites for the IBD and PST methods. The MATLAB source code, example data, and a standalone application can be freely downloaded from: http://www.mcu.edu.tw/department/biotec/ en_page/PSTReader/index.htm. 


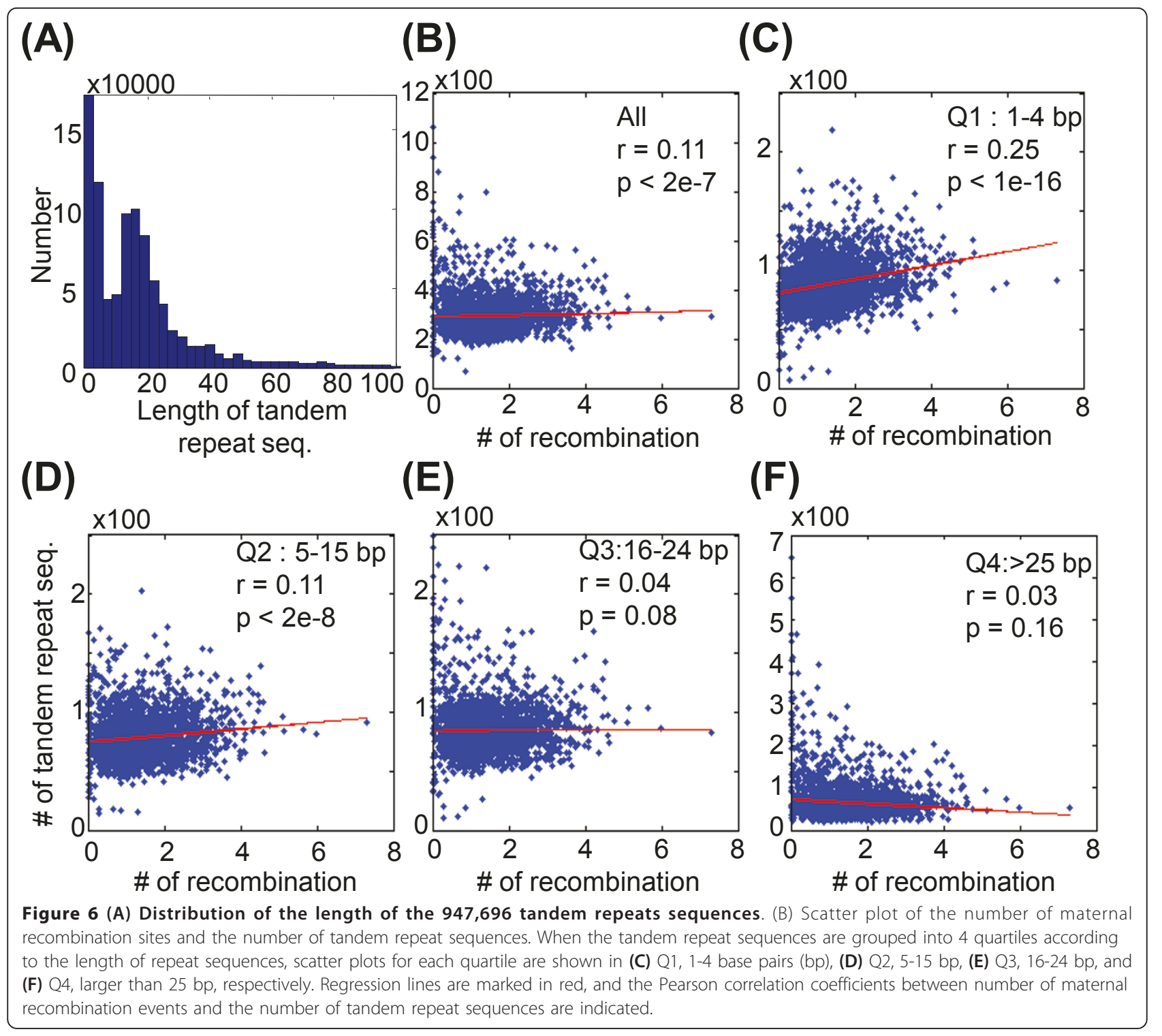

\section{GMRCL Dataset}

In this study, a set of the Affymetrix Genome-Wide Human SNP array 6.0 (GMRCL dataset) consisting of 900 K SNP markers was used as a template. DNA was extracted from blood collected in a study that was approved by the Chang Gung Memorial Hospital Institute Review Board (IRB\#99-0229B). SNP genotyping was performed using the SNP array 6.0 (Affymetrix, Santa Clara, CA, http://www.affymetrix.com) at the Genomic Medicine Research Core Laboratory (GMRCL), Chang Gung Memorial Hospital. The GMRCL dataset includes the genotypes of an anonymous family consisting of the paternal/maternal grandfather, paternal/maternal grandmother, father, mother and two children. The identitydelinked SNP genotypes and pedigree information for each member can be downloaded from http://www.mcu. edu.tw/department/biotec/en_page/PSTReader/index. htm.

\section{GSE6754 Dataset}

The GSE6754 dataset was downloaded from the Gene Expression Omnibus (GEO), and contains information from 6,971 Affymetrix GeneChip Human Mapping $10 \mathrm{~K}$ 2.0 Arrays. Data from parental and sibling genotypes are available for 1,168 families in this dataset. To increase analytic accuracy, we excluded samples with genotyping call rates less than $90 \%$, those lacking pedigree information, and individuals with chromosomal abnormalities (n = 22) [28]. The remaining 3,864 arrays of 853 families (1,721 parents and 2,145 siblings) were included in the 
PST analysis of recombination events in human meiosis. The details on individual, families, and pedigrees are provided in Additional file 5.

\section{Mapping of the recombination sites, repetitive elements and tandem repeat sequences}

The recombination sites and repetitive elements were mapped using the hg18 (NCBI Build 36) human reference assembly. The classes and characters of major repetitive elements were downloaded from RepeatMasker [24], and the tandem repeat sequences were identified using the Tandem Repeats Finder program [26]. Correlations between recombination sites and repetitive elements or tandem repeat sequences were analyzed with MATLAB (version 7.9). To assess the distribution and correlation between recombination sites and repetitive elements or tandem repeat sequences, we calculated the number of recombination sites (or repetitive elements or tandem repeat sequences) using a window width set to $1 \mathrm{Mb}$. We divided the human genome into 2765 bins of $1 \mathrm{Mb}$ each and determined the number of recombination sites in each bin. The distance for each $1 \mathrm{Mb}$ window was calculated based on SNP positions according to the Affymetrix data, assuming a constant crossover rate between two adjacent SNP markers. To calculate the correlation coefficients between the recombination in GSE6754 map, Icelandic map and Marshfield map, we divided the human genome into 2765 bins of $1 \mathrm{Mb}$ each and determined the number of recombination sites in each bin, as described above.

\section{Additional material}

Additional file 1: Calling schema. Tables with calling schema for analyzing meiosis, identity by descent (IBD) and parent-sibling tracing (PST).

Additional file 2: Paternal recombination site along the chromosomes. The paternal recombination site of child 1 and 2 of GMRCL dataset $(\mathrm{CH} 1$ and $\mathrm{CH} 2$, defined in Figure 1) along chromosomes are demonstrated in figures by the identity by descent (IBD) and parentsibling tracing (PST) methods.

Additional file 3: Distribution of recombination events. Figures illustrating the distribution of the 2,145 paternal and 2,145 maternal recombination events in human for each chromosome.

Additional file 4: Correlation between tandem repeats sequences and paternal recombination sites. Distribution of the length of the tandem repeats sequences and scatter plot of the number of paternal recombination sites with the tandem repeats sequences.

Additional file 5: Detailed information of GSE6754 dataset. Family ID, individual ID and the pedigree relative of the analyzed 3864 samples which were downloaded from GEO, GSE 6754.

\section{Abbreviations}

PST: parent-sibling tracing; IBD: identity by descent; IBS: identity by state; STRP: simple tandem repeat polymorphisms; SNP: single nucleotide polymorphisms.

\section{Acknowledgements}

This study was supported by grants: NSC 97-2320-B-130-001-MY2 (to YS Lee), NSC 98-3112-B-001-027 from the National Research Program for Genomic Medicine (to YS Lee and CH Chen); DOH99-TD-C-111-006 (to A Chao and TH Wang) and DOH99-TD-I-111-TM013 (to TH Wang) from the Department of Health, Taiwan; and CMRPG340463 (to TH Wang) from the Chang Gung Medical Foundation. The authors wish to thank Dr. Chi-Nue Tsai (Chang Gung University) and Dr. Shih-Tien T. Wang of Children's Hospital of Wisconsin, Milwaukee, for helpful discussion.

\section{Author details}

'Department of Biotechnology, Ming Chuan University, Tao-Yuan, Taiwan. ${ }^{2}$ Genomic Medicine Research Core Laboratory (GMRCL), Chang Gung Memorial Hospital, Tao-Yuan, Taiwan. ${ }^{3}$ Department of Obstetrics and Gynecology, Lin-Kou Medical Center, Chang Gung Memorial Hospital and Chang Gung University, Tao-Yuan, Taiwan. ${ }^{4}$ Institute of Statistical Science, Academia Sinica, Taipei, Taiwan. ${ }^{5}$ Department of Obstetrics and Gynecology, White Memorial Medical Center, Los Angeles, CA, USA.

\section{Authors' contributions}

YSL, AC, SMW and THW designed the study and prepared the manuscript. YSL, TC and CHC carried out the statistical analysis. YSL and THW carried out the Affymetrix microarray experiments, obtained the clinical materials and analyzed clinical information. All authors read and approved the final manuscript.

\section{Competing interests}

The authors declare that they have no competing interests.

Received: 9 December 2010 Accepted: 26 August 2011

Published: 26 August 2011

\section{References}

1. Alberts B, Johnson A, Lewis J, Raff M, Roberts K, Walter P: Molecular Biology of THE CELL. New York: Garland Science; 52008.

2. Martinez-Perez E, Colaiacovo MP: Distribution of meiotic recombination events: talking to your neighbors. Curr Opin Genet Dev 2009, 19:105-112.

3. Parvanov ED, Petkov PM, Paigen K: Prdm9 controls activation of mammalian recombination hotspots. Science 2010, 327:835.

4. Baudat F, Buard J, Grey C, Fledel-Alon A, Ober C, Przeworski M, Coop G, de Massy B: PRDM9 is a major determinant of meiotic recombination hotspots in humans and mice. Science 2010, 327:836-840.

5. Myers S, Bowden R, Tumian A, Bontrop RE, Freeman C, MacFie TS, McVean $G$, Donnelly P: Drive against hotspot motifs in primates implicates the PRDM9 gene in meiotic recombination. Science 2010, 327:876-879.

6. Coop G, Wen X, Ober C, Pritchard JK, Przeworski M: High-resolution mapping of crossovers reveals extensive variation in fine-scale recombination patterns among humans. Science 2008, 319:1395-1398.

7. Fledel-Alon A, Wilson DJ, Broman K, Wen X, Ober C, Coop G, Przeworski M: Broad-scale recombination patterns underlying proper disjunction in humans. PLoS Genet 2009, 5:e1000658.

8. Kong A, Thorleifsson G, Gudbjartsson DF, Masson G, Sigurdsson A Jonasdottir A, Walters GB, Gylfason A, Kristinsson KT, Gudjonsson SA, et al: Fine-scale recombination rate differences between sexes, populations and individuals. Nature 2010, 467:1099-1103.

9. Buard J, de Massy B: Playing hide and seek with mammalian meiotic crossover hotspots. Trends Genet 2007, 23:301-309.

10. Tease $C$, Hulten MA: Inter-sex variation in synaptonemal complex lengths largely determine the different recombination rates in male and female germ cells. Cytogenet Genome Res 2004, 107:208-215.

11. Brown PW, Judis L, Chan ER, Schwartz S, Seftel A, Thomas A, Hassold TJ: Meiotic synapsis proceeds from a limited number of subtelomeric sites in the human male. Am J Hum Genet 2005, 77:556-566.

12. Lynn A, Ashley T, Hassold T: Variation in human meiotic recombination. Annu Rev Genomics Hum Genet 2004, 5:317-349.

13. Jeffreys AJ, Murray J, Neumann R: High-resolution mapping of crossovers in human sperm defines a minisatellite-associated recombination hotspot. Mol Cell 1998, 2:267-273. 
14. Sun F, Trpkov K, Rademaker A, Ko E, Martin RH: Variation in meiotic recombination frequencies among human males. Hum Genet 2005, 116:172-178

15. Cheung VG, Burdick JT, Hirschmann D, Morley M: Polymorphic variation in human meiotic recombination. Am J Hum Genet 2007, 80:526-530.

16. Kong A, Gudbjartsson DF, Sainz J, Jonsdottir GM, Gudjonsson SA, Richardsson B, Sigurdardottir S, Barnard J, Hallbeck B, Masson G, et al: A high-resolution recombination map of the human genome. Nat Genet 2002, 31:241-247.

17. Matise TC, Sachidanandam R, Clark AG, Kruglyak L, Wijsman E, Kakol J, Buyske S, Chui B, Cohen P, de Toma C, et al: A 3.9-centimorgan-resolution human single-nucleotide polymorphism linkage map and screening set. Am J Hum Genet 2003, 73:271-284.

18. Roberson ED, Pevsner J: Visualization of shared genomic regions and meiotic recombination in high-density SNP data. PLOS One 2009, 4:e6711.

19. Ting JC, Roberson ED, Currier DG, Pevsner J: Locations and patterns of meiotic recombination in two-generation pedigrees. BMC Med Genet 2009, 10:93.

20. Chowdhury R, Bois PR, Feingold E, Sherman SL, Cheung VG: Genetic analysis of variation in human meiotic recombination. PLoS Genet 2009, 5:e1000648.

21. Barrett T, Troup DB, Wilhite SE, Ledoux P, Rudnev D, Evangelista C, Kim IF, Soboleva A, Tomashevsky M, Edgar R: NCBI GEO: mining tens of millions of expression profiles-database and tools update. Nucleic Acids Res 2007, 35:D760-765.

22. Szatmari P, Paterson AD, Zwaigenbaum L, Roberts W, Brian J, Liu XQ, Vincent JB, Skaug JL, Thompson AP, Senman L, et al: Mapping autism risk loci using genetic linkage and chromosomal rearrangements. Nat Genet 2007, 39:319-328.

23. Broman KW, Murray JC, Sheffield VC, White RL, Weber JL: Comprehensive human genetic maps: individual and sex-specific variation in recombination. Am J Hum Genet 1998, 63:861-869.

24. Jurka J, Smit AFA: "Reference collections of human and rodent repetitive elements". Co-editor of the mammalian databases 1994 [http://www.girinst. org/].

25. Kuhn RM, Karolchik D, Zweig AS, Wang T, Smith KE, Rosenbloom KR, Rhead B, Raney BJ, Pohl A, Pheasant M, et al: The UCSC Genome Browser Database: update 2009. Nucleic Acids Res 2009, 37:D755-761.

26. Benson G: Tandem repeats finder: a program to analyze DNA sequences. Nucleic Acids Res 1999, 27:573-580.

27. Myers S, Bottolo L, Freeman C, McVean G, Donnelly P: A fine-scale map of recombination rates and hotspots across the human genome. Science 2005, 310:321-324

28. Lee YS, Chao A, Chao AS, Chang SD, Chen CH, Wu WM, Wang TH, Wang HS: CGcgh: a tool for molecular karyotyping using DNA microarray-based comparative genomic hybridization (array-CGH). $J$ Biomed Sci 2008, 15:687-696.

doi:10.1186/1471-2164-12-434

Cite this article as: Lee et al:: Analysis of human meiotic recombination events with a parent-sibling tracing approach. BMC Genomics 2011

$12: 434$

\section{Submit your next manuscript to BioMed Central and take full advantage of:}

- Convenient online submission

- Thorough peer review

- No space constraints or color figure charges

- Immediate publication on acceptance

- Inclusion in PubMed, CAS, Scopus and Google Scholar

- Research which is freely available for redistribution 